\title{
Robert Grosseteste and his Treatise on Lines, Angles and Figures of the Propagation of Light
}

\section{Amelia Carolina Sparavigna'}

\author{
${ }^{1}$ Department of Applied Science and Technology, Politecnico di Torino, Torino, Italy
}

\begin{abstract}
Robert Grosseteste, an English philosopher and scientist, Bishop of Lincoln, is considered as the founder of the scientific thought in medieval Oxford. During the beginning of the XIII century he wrote several scientific papers concerning light and its propagation, where he based the description of some phenomena on the use of geometry. Here we will translate and discuss one of his scientific treatises concerning light, which is entitled De Lineis, Angulis et Figuris, seu Fractionibus et Reflexionibus Radiorum. Since to Grosseteste, the propagation of light had the main role in the creation of the world, the use of its geometry becomes a method to solve the complexity of the physical world. However, besides the use of geometry, we will find in this interesting text the description of some phenomena concerning the intensity of reflected and refracted light, which seems well-posed, even when compared with the modern Fresnel theory.
\end{abstract}

Keywords: History of Science, Medieval Science, Optics.

\section{Introduction}

Robert Grosseteste was an English scientist and philosopher of the Middle Ages. He was born into an Anglo-Norman family in the county of Suffolk in England. He became Bishop of Lincoln from 1235 AD till his death, on 9 October 1253. Considered one of the most prominent and remarkable figures of the thirteenth century, he was a man of many talents: commentator and translator of Aristotle and other Greek thinkers, philosopher, theologian, and student of Nature [1]. Besides his scholar studies that produced several treatises on theology and physics, as Bishop of Lincoln, he made a great effort on rooting out abuses of the pastoral care.

Grosseteste is considered one of the three Oxonians that played a relevant role in the revival of the studies on Optics in Western Europe [2]. After him there were Roger Bacon and John Peckham, who considered Grosseteste as an inspiration for their scientific developments. Generally, Grosseteste is described as a thinker that played a key role in the development of scientific method. A.C. Crombie $[1,3]$ describes Grosseteste as the first in the Latin West to develop an account of an experimental method in science, giving a special importance to mathematics in explaining the physical phenomena. However, this Crombie's claim that Grosseteste had used experimental methods is the subject of a considerable debate. In fact, Reference 1 is telling that the Grosseteste's method was quite different from that of a modern controlled experiment. Grosseteste, in his writings, derived his conclusions on the basis of a mix of considerations, appealing to authorities such as Aristotle or Averroes, and on everyday observations (the Latin "experimentum"). He made use of thought experiments and certain metaphysical assumptions, such as the assumption of a principle of "least action". We find this principle for instance, in the treatise entitled De Lineis, Angulis et Figuris, seu Fractionibus et Reflexionibus Radiorum, which is the subject of this paper, and in the De Iride, another of his scientific treatises on the propagation of light. The empirical observation remains the main factor for his discussion of Nature, sometimes gaining well-posed conclusions on phenomena. However, Grosseteste is far from employing an experimental method involving a controlled experiment.

Robert Grosseteste gave a relevant role to mathematics in attempting to explain the physical world. As told in [1], in his treatise On Lines, Angles and Figures, Grosseteste remarks that "the consideration of lines, angles and figures is of the greatest utility since it is impossible for natural philosophy to be known without them .... All causes of natural effects have to be given through lines, angles and figures, for otherwise it is impossible to have knowledge of the reason, the "propter quid", concerning them." [1,4] In the treatise, On the Nature of Places, a continuation of the treatise On Lines, Angles and Figures, Grosseteste remarks that "the diligent investigator of natural phenomena can give the causes of all natural effects, therefore, in this way by the rules and roots and foundations given from the power of geometry". Undoubtedly, Grosseteste saw a key role for geometry in the explanation of natural phenomena. And this emphasis on the importance of geometry and mathematics was a stimulus to thinkers 
in the Oxford of the fourteenth-century, who established the basis for the beginnings of a mathematical physics, studying in particular light and optics [3].

Grosseteste imagined the light having even a fundamental role in the creation of the world [5]: it was the light propagating in the space and dragging the matter, that originated it from a point at the beginning of times. The light is then the central subject in the Grosseteste's thought, such as the optical phenomena described by geometry. We can tell therefore that his approach to the complexity of the physical world was based on the assumption of some models, models that could be solved with geometry; however, the solutions of them are always subjected to the experience of occurring phenomena.

\section{Geometrical optics}

As previously told, Grosseteste is usually referred for his use of geometry in optics, for instance in the reflection and refraction of light. However, besides the geometry, A.C. Crombie in [6] is remarking that Grosseteste developed an analysis of the powers propagated from the natural agents. This analysis is found in four related essays written most probably in the period from 1231 to $1235 \mathrm{AD}$. The treatises on Optics are De Colore [7], De Iride [8], the De Lineis, Angulis et Figuris, and the treatise entitled De Natura Locorum. Crombie shortly commented the De Lineis, Angulis et Figuris telling that according to Grosseteste "the same power produced a physical effect in an inanimate body and a sensation in an animate one. He established rules for operation of powers: for example the power was greater for shorter and straighter the line, the smaller the incident angle, the shorter the three-dimensional pyramid or cone; every agent multiplied its power spherically. Grosseteste discussed the laws of reflection and refraction (evidently taken from Ptolemy) and their causes, and went on in De Natura Locorum to use Ptolemy's rules and construction with plane surfaces to explain refraction by a spherical burning glass" [6]. Let us remark however, that Grosseteste used the optics of Alhazen and Alkindi [9], besides that of Ptolemy.

This Crombie's discussion about the power of rays is quite stimulating to analyze the Grosseteste's treatise. Let us read it in the following section, where we are translating it from the Latin source in Reference 10. We will see that the discussion on the power of reflected and refracted rays is interesting and seems well-posed when compared with the rigorous approach given by the Fresnel reflectance formulas.

\section{Grosseteste's Lines, Angles and Figures}

The utility of considering lines, angles and figures is huge, because it is impossible to know the philosophy of Nature without them. They are valid for the entire universe and, unconditionally, for all its parts. They apply in connecting properties, such as in straight and circular motions. And they apply in action and passion (reaction), and this is so, whether in the matter or in the capacities of perception; and this is so again, whether in the sense of sight, as it is occurring, or in any other sense in the action of which it is necessary to add on other things to that which is producing the vision. Then, since we have discussed elsewhere of those things pertaining to the whole universe and to its parts in an absolute sense, and of those which are consequent to straight and circular motions, now we have to tell something concerning the universal action, when it is receiving a lower nature; this universal action is a player able of various features, so far as it happens when it is descending to act in the matter of the world; moreover, other things can be questioned, that can educate us to proceed "ad majora".

Therefore, all the causes of the natural effects must be given by lines, angles and figures, because it is impossible to know in another manner the "propter quid" in them. It is clear the following: a natural agent propagates (multiplies) its power from itself to the patient, the person or thing that undergoes some action, that is, whether it is acting on sense or on matter. This virtue is sometimes called "species", sometimes "likeness", and it is the same, in any way we call it; and the same thing is instilled in the sense and in the matter, or vice versa, when heat makes warm to the touch and gives itself to the cold body. For, it does not act through deliberation and choice; and therefore in one way it acts, whatever it is occurring, whether it is a perception or something else, animated or inanimate. But, because of the diversity of the objects of action we have different effects. Moreover, in the perception, this received power produces, in some way, a spiritual and noble effect; on the other hand, when acting on the matter, it produces a material effect, such as the sun produces, through the same power, different effects in different objects of its action, because it hardens the clay and melts the ice.

Moreover, the power produced by a natural agent can move along a shorter line, and then, it is more active, because the patient receiving it is less distant from the agent, or it can move along a longer line, and then it is less active, because the patient is more distant. And the power can come directly from the surface of an agent, or with mediation. Moreover, if it comes without mediation, it can come by a straight line, or by an oblique line. If, however, it comes by a straight line, then there is a stronger and better action, as Aristotle assumes in V Physics, because the nature acts in the shorter available way. But the straight line is the shortest of all, as he says in the same book. Similarly, a straight line has equality and no angles; but equal is 
better than unequal, as Boethius tells in his Arithmetic. And Nature acts in the possible shorter and better way, and therefore it works better on a straight line. Again, every compact power is stronger in its operations. But, the greater union and unity is in a straight line rather than in distorted line, as stated in V Metaphysics. And then an action works stronger on a straight line.

The straight line can fall either at equal angles, that is perpendicular to the surface, or at unequal angles. If it falls at equal angles, the operation is stronger for the three abovementioned reasons, because the line is shorter and equal and its power comes more uniform through it to the parts of the patient, person or thing that undergoes the action. A line, however, is falling down with equal angles on a body perpendicularly, that is with right angles, when it falls on a plane; when it falls on a concave body, it is at acute angles; but when it is falling over a sphere, it happens at angles larger than the right angle. This is shown as in the following, because, if a line is drawn passing through the center of a sphere, it makes a right angle with the line of contingency (tangency), and the line of tangency makes with the sphere on both sides the angles of contingency; then, the line falling on the sphere makes two angles with its surface, each angle larger than the right angle, being the sum of the right angle and the angle of contingency. Thus, when the power falls with angles which are not only equal, but right, then it would seem the action to be very strong, because there is complete equality and uniformity. If, however, it is not a straight line but it is a curve, nevertheless, not circular, because a natural agent does not produce its own strength according to a circle, but according to the diameter of the circle for the sake of brevity, it is manifest that such a line will have some angles. And this will not occur, as long as there is a single medium, or while there is only one body; but it is necessary that two media exist, whence in the first the power is propagated along some straight lines, and in the second along other lines.

This can happen only in two manners. First manner: that the body of the patient is dense, so as to impede the transit of power, especially in regard to our perception, and then it is said we have a reflected line, which is turning back the power. Second manner: the body the light is passing through is thin in density, which allows the propagation of power. If we have the first case, then we have the ray falling on a dense body, it falls with equal angles, that is, perpendicularly to the body, or with unequal angles, that is inclined. If we have the first manner, then it returns into itself through the same path, along which it arrived to the body. The reason of this is due to the following: the line falling on the body makes such an angle, as it is the angle made by the reflected line. And therefore it is proper that it is reflected at the same angle, upon which the ray travelled and return by the same pattern, because if it were redirected with another angle or following another pattern, turning to the left or to the right, it would be impossible that the return forms an angle equal to the angle of incidence; it would be larger or smaller. In the case that the ray is not falling perpendicularly, then it comes back along such a pattern, able to make an angle with the surface of the resisting body equal to the angle of incidence, namely, the angle which is made by the incident line with that body, for the argument already mentioned. Generally speaking, the angle of incidence and the angle of reflection are equal, and that is to be assumed now.

Since these are the two modes in which reflection may happens, it is to be understood that the reflected power into itself, because of a doubling of the power in the same place, is stronger than the reflected power in another path. Nevertheless, and this is in the essence of reflection, the action of the reflected ray is weaker, when there is the reflection in the same path, since each reflection is weakening the power, and this precise reflection, which is making the power to have a complete deviation of $180^{\circ}$ from the straight prolongation of the incident ray (that is, the direction the ray would have if it were to pass through the body), is highly weakened; and this is for the ray, which is moving on the same path on which it came from. Moreover, the path is totally contrary and opposed to the incident one, as it must be.

When we have a reflection from some bodies polished to have the same nature of the mirrors, then we have the best reflection and stronger action; but when reflection happens on rough bodies, the "species", that is, the appearance of objects to the sight, are dissipated, and the action is weak. The reason is given by Averroes, the Aristotle's Commentator, in his discussion on the sound, saying that the parts of a body surface smooth and polished, for its equality and uniformity, all together are concurring into a single action in the reflection of the species; and therefore the whole power, as it came, is reflected back from the polished body. But when the parts of a rough body are unequal, those parts protruding are reflecting the species first, and therefore there is not an agreement of the parts in a unique action, and for this reason we have a dispersion of this species randomly, and this is not a good operation.

When the reflection is obtained by means of some concave bodies, the action is stronger, than when the bodies are plane or convex, and this happens because the rays reflected by a concave surface converge together; this does not happens for the other cases. Indeed, if the medium encountered by the light is not impeding the transit of power, a ray incident at equal angles, that is perpendicularly, maintains the straight 
line and is the strongest ray. But the ray, which is incident at unequal angles, that is, inclined, deviates from the straight line that the ray had in the first medium and that it would still have if the medium were homogenous. This deviation is called refraction of rays.

The refraction is twofold: when the second medium is denser than the first, the ray is refracted to the right and passes between the prolongation of the direction of incidence and the perpendicular drawn from the point of incidence in the second medium. When the second medium is rarer, the ray is refracted to the left, receding from the perpendicular beyond the prolongation of the incident ray. And then, since these are the facts, we need to understand the reason why the power incident along a refracted line is higher that the power along a reflected ray; this happens because a refracted line little deviates from the prolongation of the incident ray, which is the strongest, and the reflected line largely deviates in the opposite direction, and then the reflection is weakening the power more than refraction.

About the power of the two modes of refraction we can tell that the power refracted to right is greater than that refracted to left, since this power, that to the right, is closer the perpendicular to the interface, whether this is the perpendicular line drawn from the incidence point or a line drawn from the agent, from which the perpendicular line and the refracted line have their origin. Besides these three fundamental lines, there is a fourth accidental line, along which an accidental and weak power moves. Which, indeed, does not come directly from an agent, but is coming from a power propagated by any of the three abovementioned lines; in such a manner, from a ray entering a window, by chance, it comes the light to all the corners of a house. However, this power is the weakest one, because it does not come directly from the agent, but it is separated from the power of the agent, in a straight line, or reflected or refracted. These facts we told about lines and angles.

About the figures, there are two kinds of them that we have to consider here. One of these is suitable for propagation of power, namely the sphere. And this happens for the following reason: every agent emanates its power spherically, since it does all around and in every direction (diameter): upwards and downwards, ahead and aback, right and left. And this is shown by the manner in which it is possible to draw a line in a certain direction from an agent located at the center, and in all directions from all the different positions, and therefore it is proper to use that spherical figure. And this is in agreement with what the Commentator (Averroes) says on the (Aristotle's) De anima. Also, wherever we put the sensor to receive, we can feel such an agent at a proper distance; however, this happens only by species or by the power coming from the agent. So the power is propagating everywhere.

Another figure, however, is required for the natural action, that is, the pyramidal one: since, if the power is coming out from a single part of the agent and ending onto another single part of the patient, and so on for all the parts of agent and patient, we always had the power from a part of the agent falling onto a sole part of the patient, and then the action will never be strong or good. However, the action is complete, when the power of the agent comes from all the points of the agent or from its whole surface to every point of the patient. But this is impossible, except under the pyramidal figure, because the power that comes from each of the parts of the agent are concurring in the cone of the pyramids and are gathered together and then they all are able to act more strongly upon the part of the patient where they are condensed.

Therefore, an infinite number of pyramids can come out from a surface of an agent, pyramids having the same basis, namely, the surface of the agent, and there are so many cones as the pyramids are, falling into different points of the medium or on all sides of the patient, and there can be an infinite number coming out from surface, some shorter some longer. However, those cones which are equal in length and size, do not have different features, because they act in the same manner, though there can be a variety of features coming from the recipient matter, inasmuch it is concerning it. But when one pyramid is shorter than another, and both are coming out by the same agent, we have a quite difficult problem to solve, that of telling whether is the cone of the shorter pyramid acting more on the patient or not.

And then, we ought to suppose that the shorter pyramid acts more, because its cone is less distant from its source, and for that reason, there is more power in it than in the longer pyramid and then the patient is more closely connected to the agent and therefore strongly altered by its power. Moreover, if the rays which are in the bulk of a shorter pyramid, that come from the right side, are prolonged besides the vertex, uninterrupted and straight, they will form smaller angles with the left beams, which are in the bulk of the pyramid, than the similar rays which are coming from a longer pyramid, as it is clear from the 21th section of first book of Euclid Geometry, and also by the common sense. And in the same way, the rays coming from the left of the pyramid, which continues beyond the vertex, uninterrupted and straight, are closer to the rays of the right side in the bulk of the pyramid, than the consimilar rays of a longer pyramids. Then, because any congregation or union is 
more active, the cone of a short pyramid acts more and alters the patient more than a longer cone. However, we could object rationally that, when from all the surface of an agent the power is coming in a longer pyramid, we have there more power, because the cone is more acute than that of a shorter pyramid, and all the power is condensed for a greater operation, and there is also to add the following, that the rays of a longer pyramid are close to the rays of the agent, those lines which are drawn perpendicularly from the ends of the diameters of the agent, and then they are stronger, because the perpendicular progression is the strongest: it can be said that these reasons seems rather well posed, and they could be, if there were not the strongest reasons to the contrary, which we have mentioned previously. This is the end of the treatise by a Lincolnian on the reflections and refractions of rays.

\section{Comments to the Grosseteste's text}

The treatise has a strong Incipit: let us therefore report, after the Grosseteste's words what A.G. Padgett is telling about Grosseteste in [11]. "Even as he translated and interpreted Aristotle, Grosseteste placed Aristotelian natural philosophy in a broader Christian and Neo-platonic world view. ... he was committed to a natural philosophy based upon mathematics. This emphasis derived from Platonic and Pythagorean traditions, as mediated to him through Patristic authors like Augustine. A mathematical natural philosophy is demonstrated in a number of his works, particularly works on astronomy, light, and in his treatise on geometry, De Lineis, Angulis et Figuris." As we have seen in reading this treatise, it is not only a treatise on geometry, as told by Padgett, but on the geometry applied to light propagation. Padgett continues in [11] telling that in the incipit of the treatise, Grosseteste defends his mathematical approach to natural philosophy. "Notice that Grosseteste wants to use geometry, which was long a key tool of astronomers, within natural philosophy. This is a decisive step in the history of Western science, although Grosseteste was not alone in making it." [11]

Continuing our reading of the Grosseteste's treatise, we find that the philosopher is proposing a universal action descending in the lower world, according to an Aristotelian view of the universe [12]. And this action can have material and spiritual effects, for instance helping a person to achieve some intellectual results. In the Latin text, we find also that Grosseteste refers to the "species". "Species" in Latin means "seeing", "view", "look", or "sight", but also "external appearance", "general outline" or shape. Then the "species" is that feature of the power of light which allows perceiving the shape of an object.

In another treatise written by Grosseteste, the De Iride [8], we found the "quid", that is the effect, or the phenomenon, the physics needs to describe, and the "propter quid", which is instead an answer given by the research, on the causes of the phenomenon. And here Grosseteste is telling that without the geometry we are not able to find the "proper quid". As previously told, in the first part of his treatise, Grosseteste is claiming the necessity to use mathematics and geometry to explain physics.

How is the light moving? According to Grosseteste, it is a principle of least action to rule it. We can repeat also what Grosseteste is telling in De Iride [8]: "And the same tells us that principle of the philosophy of nature, namely, that every action of the nature is well established, most ordinate, in the best and shortest manner, as it is possible." This principle is aiming to find a figure in the complexity of the world.

After stating this principle, Grosseteste discusses what is happening when light falls onto a surface, that is, he is discussing about illumination. We know that illumination is following a cosine law, a geometric relationship between the illuminance of a surface and the angle of incidence of the illuminating rays $[13,14]$. The observed maximum of illuminance is therefore obtained for normal incidence, as Grosseteste is telling in his text. For what is concerning the angles, let us stress that in the Grosseteste's discussion, we can find that the Medieval scientists regarded "contingent angles", that is the angles of tangency, as having a finite magnitude [12]. Therefore the contingent angle is different if it is of a convex or concave surface.

The Grosseteste's treatise is also discussing the reflection and refraction of light as told by its title. We find here that Grosseteste is explaining that to bend the light we need several different media, so that at the interfaces the ray is broken with certain angles. This is discussed in the De Iride [8] too, where we find even a law of refraction, which tells that the angles of refraction are one half the angles of incidence.

In the Latin text, Grosseteste is telling that the power "multiplies" along a straight line. Therefore, he imagined the light propagating by multiplying itself [15], and here, in translating the his words, we rendered this propagation like that proposed by Huygens for the waves. In 1678, Christiaan Huygens considered that each point of a luminous wavefront could be the source of a spherical wavelet. The sum of these wavelets determines the new propagated wavefront. He assumed that the secondary waves travelled only in the forward direction. And then the light is "generating" itself, in the sense of propagation. Probably, Grosseteste imagined a similar mechanics, without waves however.

Grosseteste is also discussing the "doubling" of the 
power (in the Latin text, Grosseteste is proposing a "gemination"). A possible interpretation can be the following: let us consider a ray of light normally incident on a surface and the reflected ray, radiated back into the half-space of the incident ray. It means that in the volume occupied by these rays, which is the same, we have a "doubling", a superposition of power. In any other case, that is, when the incidence is oblique, a certain volume of the space can be occupied just by the incident or by the reflected ray. And therefore, to Grosseteste the power of the reflected rays is depending on the angle of incidence: his description is in agreement with the fact that the light falling at an angle on a surface tends to be increasingly reflected as the angle of incidence increases, and the transmission reduced. For a normal incidence in fact, we have the largest amount of transmitted power and, of course, the smallest amount of reflected power.

Usually, the behavior of the reflected light with the angle of incidence is studied with the Maxwell's equations, allowing to derive the Fresnel equations (see for instance, the Fresnel laws of reflection as discussed by a chapter in the first volume of the Feynman Lectures on Physics), which can be used to predict how much of the light is reflected and refracted. On a specular reflection then, we have that the fraction of the reflected light increases with the increase of the angle of incidence. Let us remember that the Fresnel reflectance for metals and dielectric materials is very different. For a metal such as aluminum, the reflectance is always above the $85 \%$. For a glass having a refractive index of $n=1.5$, the reflectance is of only $4 \%$ at normal incidence, but $100 \%$ at grazing. "This effect, in fact, is what makes polished metals look like metal, and polished glasses not look that way. It's also why it's hard to comb your hair in a shop window; you are looking at the angle of minimum reflectance.” [16]

In the Grosseteste's text, we can find also that he is distinguishing between specular and Lambertian surfaces. Very interesting is the fact that Grosseteste is using an analogy with the sound waves, telling that Averroes, the Aristotle's Commentator, studied the sound propagation and the role of irregular surfaces in break down the reflection of it. The treatise continues with an analysis of emitted and received power, based on pyramids and solid angles; it ends with proposing and solving a question concerning the power of small and large solid angles.

\section{Conclusions}

In this paper we have proposed a translation of one of the Grosseteste's treatise on the propagation of light, entitled De Lineis, Angulis et Figuris, seu Fractionibus et Reflexionibus Radiorum. As emphasized in the previous section, the discussion of Grosseteste about the power of the reflected and refracted light is in qualitative agreement with the Fresnel formulae of reflection and refraction. The discussion of the illumination of surfaces is quite good too.

In the Grosseteste's treatises, diagrams or formulae do not exist; however, it is quite clear the role of geometry. As told in the Reference 1, Grosseteste gave a special importance to mathematics in attempting to provide scientific explanations of the physical world. The same reference tells also that at the basis of the reasoning on light, there was Grosseteste's view that natural agents act by the multiplication of their power or species, a view developed further on by Roger Bacon. However, let us note that if we consider the "multiplication" as propagation, this could be a sort of propagation of light as Huygens imagined several years after. "Grosseteste holds that the intensity of operation of the natural agent will be a matter of its distance from what it acts upon, the angle at which it strikes it, and the figure in which it multiplies its operation, this being either a sphere or cone. He establishes certain rudimentary rules to this effect, such as that the shorter the distance, the stronger the operation", it is told in [1]. However, as we have seen from reading Grosseteste's treatise, some observations on the power of transmitted and reflected light are more than rudimental, because probably he experimented about them.

Let us conclude that Robert Grosseteste aimed to solve the complexity of the world by using geometry and mathematics applied to experimental observations. He saw the natural philosophy, that is the physics, based upon them, and stressed this theoretical approach in several of his treatises. To the Western Europe of the Middle Ages, the Grosseteste's approach was a decisive step towards the modern science.

\section{References}

[1] N. Lewis, Robert Grosseteste. The Stanford Encyclopedia of Philosophy http://plato.stanford.edu/entries/grosseteste/,

[2] G. ten Doesschate, Oxford and the revival of Optics in the thirteenth century, Vision Review, Volume 1, 1962, Pages 313-342.

[3] A.C. Crombie, Grosseteste's Position in the History of Science, in Robert Grosseteste: Scholar and Bishop, ed. Daniel A. Callus, Oxford: Clarendon Press, 1955, Pages 98120.

[4] L. Baur, editor, Die Philosophischen Werke des Robert Grosseteste, Bischofs von Lincoln, Beiträge zur Geschichte der Philosophie des Mittelalters, Münster: Aschendorff Verlag, 1912.

[5] A.C. Sparavigna, From Rome to the Antipodes: the Medieval Form of the World, arXiv, 2012, http://arxiv.org/abs/1211.3004

[6] A.C. Crombie, Science. Art and Nature in Medieval and Modern Thought, Continuum International Publishing Group, Aug 2, 2003.

[7] A.C. Sparavigna, Robert Grosseteste's Colours, arXiv, 2012, History and Philosophy of Physics, arXiv:1212.6336, http://arxiv.org/abs/1212.6336 
[8] A.C. Sparavigna, Translation and Discussion of the De Iride, a Treatise on Optics by Robert Grosseteste, arXiv, 2012, History and Philosophy of Physics, arXiv:1211.5961, http://arxiv.org/abs/1211.5961

[9] Late Medieval and Early Modern Corpuscular Matter Theories, edited by C.H. Lüthy, J.E. Murdoch and W. Royall Newman, Brill, 2001, Page 190.

[10] The Latin text is available at "The Electronic Grosseteste", http://www.grosseteste.com/

[11] A.G. Padgett, The Roots of the Western Concept of the "Laws of Nature": From the Greeks to Newton, Perspectives on Science and Christian Faith, Volume 55, Number 4, December
http://www.asa3.org/ASA/PSCF/2003/PSCF1203Padgett.pdf/

[12] E. Grant, A Source Book in Medieval Science, Harvard University Press, 1974.

[13] P. Tregenza and D. Loe, The Design of Lighting, Taylor \& Francis, 1998

[14] J.L. Lindsey, Applied Illumination Engineering, The Fairmont Press, Inc., 1997.

[15] C.C. Riedl, Robert Grosseteste, On Light, Marquette University Press, Milwaukee, Wisconsin, 1942.

[16] S.H.

Westin http://www.graphics.cornell.edu/ westin/misc/fresnel.html 